\title{
BIODIVERSITY CONSERVATION THROUGH RELIGIOUS BELIVES IN SACRED GROVES OF JABALPUR FOREST DIVISION (MADHYA PRADESH)
}

\author{
RUBY RAI DUGGAL ${ }^{1}$, S. K. MASIH ${ }^{2} \&$ R. P. MISHRA ${ }^{3}$ \\ ${ }^{1}$ Department of Postgraduate Studies and Research in Biological Science \\ Rani Durgavati University, Jabalpur, Madhya Pradesh, India \\ ${ }^{2}$ State Forest Research Institute, Polipathar, Jabalpur, Madhya Pradesh, India \\ ${ }^{3}$ Department of Postgraduate Studies and Research in Biological Science \\ Rani Durgavati University, Jabalpur, Madhya Pradesh, India
}

\begin{abstract}
Sacred Groves are the one form of nature worship, which is considered as "Sacred Natural Sites". Significant tradition of nature worship is provides protection to patches of forests dedicated to deities and ancestral spirits by the tribal and forest dwellers. The survey work was carried out from 2013-2016 in Jabalpur forest division, to assess the role of sacred groves in biodiversity conservation. Historical background, ethnic belief and the socio-cultural importance about sacred groves were collected from each sacred grove. Total 105 sacred groves had been identified from the seven forest ranges of Jabalpur forest division. The 129 plant species belonging to 56 families and 105 genus are conserved in these sacred groves, due to their socio-religious importance. According to their mode of use such as during different festivals and religious ceremonies are discussed in this paper, in detail.

KEYWORDS: Historical Background, Ethnic Beliefs \& Socio Cultural
\end{abstract}

Received: Mar 31, 2017; Accepted: Apr 25, 2017; Published: May 15, 2017; Paper Id.: IJASRJUN201723

\section{INTRODUCTION}

Sacred groves are forest fragments of varyaing sizes, communally protected in the name of local gods and goddess. Hunting and logging are strictly prohibited within these patches. Indian sacred groves have pre-Vedic origin. Most of them are associated with indigenous and tribal communities, who most believe in divinity of nature and natural resources. Sacred groves in India are sometimes associated with temples, monasteries, shrines and burial grounds. Patnaik \& Pandey (1998) defined sacred groves as culturally a living place of the deities and spirits of the village, which protects them from calamities and bring prosperity and a patch of pristine forest, having well conserved biodiversity. In return, the villagers regularly propitiate them by means of rituals, sacrifice on festivals or seasons designated for the same.

These groves are scattered all over the country, and are referred by different names in different states. According to Sukumaran \& Jeeva (2008), sacred grove is a small area of land with a particular type of tree grown on it, which is considered to be holy by the local human community. Sacred groves are groups of trees or patches of vegetation protected by the local people through religious and cultural practices, evolved to minimize distraction. The significant tradition of nature worship is that of providing protection to patches of forests dedicated to deities or ancestral spirits. These vegetation patches have been designated as sacred groves (Patel \& Patel, 2012).

Jabalpur is a district in Madhya Pradesh state. It lies between the latitudes $22^{\circ} 37^{\prime \prime} \mathrm{N}$ to $22^{\circ} 50^{\prime \prime} \mathrm{N}$ and 
longitudes of $79^{\circ} 20^{\prime \prime}$ to $80^{\circ} 34^{\prime \prime}$ East. The area of the district is $5211 \mathrm{~km}^{2}$. It is located in the watersheds of the Narmada and the Son rivers. According to the 2011 census, the total population of the district is 2,463,289. According to Indian State of Forest Report (ISFR, 2015), the total forest area of the Jabalpur forest division is 117000 ha, out of which, very dense forest, moderately dense forest, open forest and scrub are 3600 ha, 51400 ha, 62000 ha and 15400 ha, respectively. As per the Classification of the forest area of India (Champion and Seth, 1968), the forest area of Jabalpur forest division is classified under the following classes:-

- 5AC1b: Southern tropical dry deciduous teak forests

- 5AC3: Southern tropical dry deciduous mixed forests

According to Working Plan of Jabalpur Forest Division, M. P. Forest Department (Sinha, 2005-06 to 2014-15), the forest division is divided into seven forest ranges, namely Panagar, Kundam, Sihora, Jabalpur, Patan, Sapporo, and Bargi. These seven ranges are having 81 beats and 345 compartments.

\section{METHODOLOGY}

Detailed field survey of existing sacred groves in the Jabalpur forest Division of Madhya Pradesh was carried out from 2013 to 2016.

Each sacred grove had been visited individually and recorded by GPS location, photographs and detail information regarding its forest range, name, type, management class, area (in $\mathrm{m}^{2}$ ), age class (existence), deity of the grove, other associated deity (if any), offerings, day of worship and plant diversity of the grove. Sacred groves are frequently visited on major festivals celebrated there.

All plant species recorded from various sacred groves were identified and arranged family species wise, with the help of "Flora of British India” Hooker's (1872-1897); “The Forest Flora of North-West and Central India” Brandis \& Stewart (1874); "Flora of Tamil Nadu” Nair \& Henry (1983). "Flora of Jabalpur" Oommachan \& Shrivastava (1996); "Supplement to the Flora of Madhya Pradesh" Khanna, Kumar, Dixit \& Singh (2001); "Flora of Madhya Pradesh Vol. - 1" Verma, Balakrishan \& Dixit (1993); "Flora of Madhya Pradesh Vol. - 2" Mudgal, Khanna \& Hajra (1997); and "Flora of Madhya Pradesh Vol. - 3” (Singh, Khanna, Mudgal \& Dixit (2001).

\section{RESULTS AND DISCUSSIONS}

\section{Sacred Groves}

During the study, all seven ranges, namely Panagar, Kundam, Sihora, Jabalpur, Patan, Sapporo, and the barge were visited to identify the existing sacred groves. Total 105 sacred groves have been identified from different beats and forest compartments of these ranges. The highest number of sacred groves (24) has been identified from Kundam forest ranges, whereas the lowest number of sacred groves (3) identified from a Patan forest range. The numbers of identifies sacred groves in different ranges are shown in Table $\mathbf{1 .}$

Table 1: No. of Identified Sacred Groves in different Ranges of Jabalpur Forest Division

\begin{tabular}{|c|l|c|}
\hline S. No. & \multicolumn{1}{|c|}{$\begin{array}{c}\text { Name of Forest } \\
\text { Range }\end{array}$} & $\begin{array}{c}\text { No. of Identified } \\
\text { Sacred Groves }\end{array}$ \\
\hline 1 & Bergi & 23 \\
\hline 2 & Jabalpur & 20 \\
\hline
\end{tabular}




\begin{tabular}{|c|l|c|}
\hline \multicolumn{2}{|c|}{ Table 1: Contd., } \\
\hline 3 & Kundam & 24 \\
\hline 4 & Panager & 12 \\
\hline 5 & Patan & 3 \\
\hline 6 & Sehpura & 12 \\
\hline 7 & Sihora & 11 \\
\hline \multicolumn{2}{|c|}{ Total } & $\mathbf{1 0 5}$ \\
\hline
\end{tabular}

\section{Plants of Socio-Religious Importance}

During the survey work on existing sacred groves of Jabalpur forest division, an inventory was made of plant species, having sacred and religious importance. Total 129 plant species belonging to 56 families and 105 genuses were catalogued, out of which, some of them are tree, shrub, herb, climber and grass categories. The list of such recorded plant species is given in Table 2 with botanical name, local name, part used and name of the festival and socio-religious occasion and socio-religious importance of the species. A number of plant parts used for various religious occasions are shown in Figure 1.

Table 2: Plants of Socio-Religious Importance

\begin{tabular}{|c|c|c|c|c|}
\hline S. No. & Botanical Name & Local Name & Part Used & Socio-Religious Occasion \\
\hline 1 & Acacia catechu & Kattha, Khair & Whole plant & Agni deo pooja and Hawans \\
\hline 2 & Achyranthes aspera & $\begin{array}{l}\text { Apamarg, } \\
\text { Chirchira }\end{array}$ & Branch & Various faste \\
\hline 3 & Actiniopteris radiata & Morpankhi ghans & Whole plant & Harchat pooja \\
\hline 4 & Aegle marmelos & Bel & Leaves, fruits & $\begin{array}{l}\text { Savwan somwar, Maglagauri, } \\
\text { Shiveratri pooja, Krishna and } \\
\text { janmashtami }\end{array}$ \\
\hline 5 & Annona reticulata & Ram phal & Whole plant & Worshiped as sacred plant \\
\hline 6 & Annona squamosa & Sheeta phal & Fruit & $\begin{array}{l}\text { Durga, Lakshmi, Saraswati, } \\
\text { Dashera and Gyaras pooja }\end{array}$ \\
\hline 7 & Anthocephalus cadamba & Kadam & Whole plant & $\begin{array}{l}\text { Krishna janmashtami, Gudi } \\
\text { padwa }\end{array}$ \\
\hline 8 & Araca catechu & Supari & Fruit & Mangala Gauri pooja \\
\hline 9 & Avena sterilis & Jawa & Branch, flower & $\begin{array}{l}\text { Basant Panchami, Janki navmi } \\
\text { and Shuki oaksh }\end{array}$ \\
\hline 10 & Azadirachta indica & Neem & Whole plant & $\begin{array}{l}\text { Gudi padwa, Shiva pooja, Santaan } \\
\text { saptmee }\end{array}$ \\
\hline 11 & Bauhinia purpurea & Keolar & Flower & Shiva pooja \\
\hline 12 & Bauhinia vahlii & Mahul & Leaves & Poojas \\
\hline 13 & Bauhinia veriegate & Kachnar & Flower & Shiva pooja, Santaan saptmee \\
\hline 14 & Bixa orellana & Latkan/Sindoor & Fruit & Various poojas \\
\hline 15 & Boerhavia diffusa & $\begin{array}{l}\text { Punarnava/Pathar- } \\
\text { chatta }\end{array}$ & Whole plant & Worshiped as sacred plant \\
\hline 16 & Brassica copastris & Sarson & Seed & Poornima, akshay tritiya \\
\hline 17 & Bridelia retusa & Kasai & Whole plant & Worshiped as sacred plant \\
\hline 18 & Buchanania lanzan & Achar & Seed, branch & Various pooja \\
\hline 19 & Butea monosperma & Palash & Whole plant & $\begin{array}{l}\text { Manglagauri, Ganesh pooja, } \\
\text { Navratri, Shiv pooja. Poornima } \\
\text { akshay tritiya and Hal Shasthi }\end{array}$ \\
\hline 20 & Calatropis gigaintia & Aak & Flower, leaves & $\begin{array}{l}\text { Manglagauri, Ganesh pooja, } \\
\text { Navratri, Shiv pooja, Holi }\end{array}$ \\
\hline 21 & Calatropis procera & Aak white, Madar & Flower, leaves & $\begin{array}{l}\text { Death occasion, Shivratri, } \\
\text { Bajarngbali, Manglagauri, } \\
\text { Navratri, Ganesh pooja }\end{array}$ \\
\hline 22 & Cannabis sativa & Bhang & Whole plant & $\begin{array}{l}\text { Shivaratri pooja, holi, Navratri } \\
\text { and for black magic }\end{array}$ \\
\hline
\end{tabular}




\begin{tabular}{|c|c|c|c|c|}
\hline \multicolumn{5}{|c|}{ Table 2: Contd., } \\
\hline 23 & Capparis sepiaris & Andi, aundi & Whole plant & Holi pooja \\
\hline 24 & Capsicum annum & Mirchi & Fruit & Prosparity decoration \\
\hline 25 & Carica papaya & Papita & Fruit & Various poojas \\
\hline 26 & Carissa carandas & Karonda & Fruit & Various poojas \\
\hline 27 & Cassia fistula & Amaltas & Whole plant & Worshiped as sacred plant \\
\hline 28 & Catharanthus roseus & Sadasuhagan & Flower & Various poojas \\
\hline 29 & Cicer arietinum & Chana & Fruit & Shivratri, Bajarngbali pooja \\
\hline 30 & Citrus aurantifolia & Aterra neebu & Fruit & $\begin{array}{l}\text { Navratri, Shivratri poojas and for } \\
\text { black magic }\end{array}$ \\
\hline 31 & Citrus medica & Neembu & Fruit & $\begin{array}{l}\text { Navratri, Shivratri poojas and for } \\
\text { black magic }\end{array}$ \\
\hline 32 & Cleome viscosa & Hulhul & Whole plant & Poojas \\
\hline 33 & Clitoria ternatea & Aprajita & Whole plant & $\begin{array}{l}\text { Prosparity decoration, Marriage } \\
\text { ceremony }\end{array}$ \\
\hline 34 & Coccus nucifera & Nariyal & Fruit & $\begin{array}{l}\text { Various poojas specially Ganesh } \\
\text { and Mahalaxmi pooja }\end{array}$ \\
\hline 35 & Curcuma angustifolia & Tikhur & Rhizome & During faste \\
\hline 36 & Curcuma caesia & Kali haldi & Whole plant & Siddhi pooja \\
\hline 37 & Curcuma domestica & Jangli haldi & Fruit & Various poojas \\
\hline 38 & Curcuma longum & Haldi & Fruit & Applied to the forehead of gods \\
\hline 39 & Cynodon dactylon & Doob & Whole plant & $\begin{array}{l}\text { Hal Shasthi, Ganesh and } \\
\text { Mahalaxmi pooja, prosparity } \\
\text { decoration }\end{array}$ \\
\hline 40 & Cyperus scariosus & Nagarmotha & Rhizome & $\begin{array}{l}\text { Rhizome powder is used as dhoop } \\
\text { during pooja. }\end{array}$ \\
\hline 41 & Dalbergia sissoo & Shisham & Whole plant & Various poojas \\
\hline 42 & Datura metel & Kala dhatura & Fruit & Shivaratri pooja \\
\hline 43 & Datura stramonium & Dhatura & Fruit & Shivaratri pooja \\
\hline 44 & Delonix regia & Gulmoher & Flower & Various poojas \\
\hline 45 & Desmodium triflorum & Teen patiya & Whole plant & Poojas \\
\hline 46 & Desmostachya bipinnata & Kush grass & Whole plant & Various religious ceremony \\
\hline 47 & Dioscorea hispida & Baichandi & Whole plant & Poojas \\
\hline 48 & Diospyros melynoxylon & Tendu & Fruit & Various pooja \\
\hline 49 & Eclipta prostrate & Bhringraj & Whole plant & Poojas \\
\hline 50 & Elaeocarpus sphaericus & Rudraksha & Fruit, leaves & $\begin{array}{l}\text { For Nakshatra shanty, to cover the } \\
\text { marriage mandap }\end{array}$ \\
\hline 51 & Elephantopus scaber & Ban tambaku & Leaves & Shivaratri pooja \\
\hline 52 & Elettaria cardimomum & Ilaychee & Fruit & Mangala Gauri pooja \\
\hline 53 & Euphorbia hirta & Dudhi & Flower & Poojas \\
\hline 54 & Evolvulus elsinoides & Sankhpushpi & Flower & Shiv pooja \\
\hline 55 & Ficus bengalensis & Bargad, Bar & Whole plant & $\begin{array}{l}\text { Vad Savitri, Bar amavashya, } \\
\text { Satyanarayan, Ganesh Chaturthi, } \\
\text { Durga pooja and to cover } \\
\text { marriage mandap }\end{array}$ \\
\hline 56 & Ficus hispida & Kagsha & Whole plant & Worshiped as sacred plant \\
\hline 57 & Ficus racemosa & Goolar & Branch & Hal Shasthi \\
\hline 58 & Ficus religiosa & Peepal & Whole plant & $\begin{array}{l}\text { Harchhath, Ganesh, Shani, Durga } \\
\text { and rishi pooja }\end{array}$ \\
\hline 59 & Ficus virens & Pakar & Whole plant & $\begin{array}{l}\text { Navratri, Anant pooja and in } \\
\text { Cremation }\end{array}$ \\
\hline 60 & Flacourtia indica & Kakai & Whole plant & Worshiped as sacred plant \\
\hline 61 & Glericidia sepium & Giripushpa & Flower & Various poojas \\
\hline 62 & Gloriosa superba & $\begin{array}{l}\text { Kalihari, } \\
\text { Kirkitchyau }\end{array}$ & Leaves, flower & $\begin{array}{l}\text { Harchat, Mahalaxmi and Anant } \\
\text { pooja }\end{array}$ \\
\hline 63 & Gossypium hirsutum & Kapas & Fruit & Poojas \\
\hline 64 & Haldinia cordifolia & Haldu & Whole plant & Worshiped as sacred plant \\
\hline
\end{tabular}




\begin{tabular}{|c|c|c|c|c|}
\hline \multicolumn{5}{|c|}{ Table 2: Contd., } \\
\hline 65 & Hibiscus rosa-sinensis & Jasoon, Gurhal & Flower & Durga pooja, Laxmi pooja \\
\hline 66 & Hiptage benghalensis & Madhavi Lata & Whole plant & Worshiped as sacred plant \\
\hline 67 & Indigofera pulchella & Neel & Whole plant & Worshiped as sacred plant \\
\hline 68 & Ipomoea batas & Sakarkand & Rhizome & $\begin{array}{l}\text { Ganesh Chaturthi vrata, Tulsi } \\
\text { vivah, Satyanarayan katha and } \\
\text { Mahalaxmi pooja }\end{array}$ \\
\hline 69 & Ixora coccinea & Rugmini, Red star & Flower & Shiv pooja \\
\hline 70 & Jasminum grandiflorum & Chameli & Flower & Many religious occasions \\
\hline 71 & Jasminum officinalis & Chadni & Flower & Various poojas \\
\hline 72 & Jasminum sambac & Bela & Flower & Many poojas \\
\hline 73 & Jatropha curcas & $\begin{array}{l}\text { Ratanjot, safed } \\
\text { arund }\end{array}$ & Flower & Many poojas \\
\hline 74 & Lannea coromandelica & Moyan & Whole plant & Worshiped as sacred plant \\
\hline 75 & Lausonia inermis & Mehendi & Leaves & $\begin{array}{l}\text { Durga pooja, Rakhshabandan, } \\
\text { Marrige ceremony }\end{array}$ \\
\hline 76 & Limonia acidissima & Kaitha & Fruit & Mahashivratri pooja \\
\hline 77 & Madhuca latifolia & Mahua & Whole plant & $\begin{array}{l}\text { Harchhath, Anant and Druga } \\
\text { pooja }\end{array}$ \\
\hline 78 & Mallotus philippensis & Sinduri & Fruit & Various poojas \\
\hline 79 & Mangifera indica & Aam, Mango & Leaves, flower & $\begin{array}{l}\text { Mangalagauri, Basant Panchami, } \\
\text { Navratri, Anant and Srikrishna } \\
\text { pooja }\end{array}$ \\
\hline 80 & Michelia champaca & Sone champa & Flower & Shivratri, Navratri pooja \\
\hline 81 & Mimusops elengi & Maulsari & Whole plant & Worshiped as sacred plant \\
\hline 82 & Mirabilis jalapa & Gulbans & Flower & Various poojas \\
\hline 83 & Mitragyna parvifolia & Mundi & Whole plant & Worshiped as sacred plant \\
\hline 84 & Morus australis & Shahtut & Fruit & Various poojas \\
\hline 85 & Murraya paniculata & madhukamini & Whole plant & Various poojas \\
\hline 86 & Musa paradisiaca & Kela & Whole plant & $\begin{array}{l}\text { Devthan pooja, Ahoeashtami, } \\
\text { Tulsi vivah and for getting child }\end{array}$ \\
\hline 87 & Nelumbium nucifera & Kamal, Lotus & Flower & $\begin{array}{l}\text { harchhat, Lakshmi, Saraswati, } \\
\text { Vishnu and Brahma pooja }\end{array}$ \\
\hline 88 & Nerium indicum & Kaner & Leaves & $\begin{array}{l}\text { Mahashivratri pooja, Cremation } \\
\text { and in Decoration }\end{array}$ \\
\hline 89 & Nyctanthes arbortristis & Harsingar & Flower & $\begin{array}{l}\text { Hartalica, Ashokashtami and } \\
\text { Anant pooja }\end{array}$ \\
\hline 90 & Ocimum americanum & Tulsi & Whole plant & Worshiped as sacred plant \\
\hline 91 & Ocsimum basilicum & Bobai & Whole plant & Makarsankrati pooja \\
\hline 92 & Pheonix sylvestre & Khajoor & Fruit & Addra nakshtra pooja \\
\hline 93 & Phyllanthus amarus & Bhui amla & Whole plant & $\begin{array}{l}\text { Aayal Ekadashi, Amlanaumi, } \\
\text { Hartalika teej, Vad Savitri Puja, } \\
\text { Bar amavashya and Laxmi pooja }\end{array}$ \\
\hline 94 & Phyllanthus emblica & Amla & Whole plant & $\begin{array}{l}\text { Aayal Ekadashi, Amlanaumi, } \\
\text { Hartalika teej, Vad Savitri Puja, } \\
\text { Bar amavashya and Laxmi pooja }\end{array}$ \\
\hline 95 & Piper betle & Pan & Leaves & $\begin{array}{l}\text { Harchhath, Mangala Gauri, Anant } \\
\text { and Druga pooja }\end{array}$ \\
\hline 96 & Pithecelobium dulce & Jangli Jalabi & Pods & Various poojas \\
\hline 97 & Plumeria rubra & Champa & Flower & Shivratri, Navratri pooja \\
\hline 98 & Pongamia pinnata & Karanj & Flower & Shivratri, Navratri pooja \\
\hline 99 & Prosopis cineraria & Shammi & Whole plant & Dasehra, Manglagauri pooja \\
\hline 100 & Psidium guajava & Amarood & Fruit & Various poojas \\
\hline 101 & Punica granatum & Anar & Fruit & Various poojas \\
\hline 102 & Putranjiea roxburghii & Putranjiwa & Whole plant & For gatting child \\
\hline 103 & Saccharum officinarum & Ganna & Whole plant & $\begin{array}{l}\text { Devthan pooja, Ahoeashtami, } \\
\text { Tulsi vivah }\end{array}$ \\
\hline
\end{tabular}




\begin{tabular}{|c|c|c|c|c|}
\hline \multicolumn{5}{|c|}{ Table 2: Contd., } \\
\hline 104 & Sacchrum spontaneum & Kans, kansa & Whole plant & Harchhath and for decoration \\
\hline 105 & Santalum album & Chandan & Stem & Various poojas \\
\hline 106 & Saraca asoca & Ashok & Whole plant & $\begin{array}{l}\text { Hartalica, Anant pooja, } \\
\text { Ashokashtami }\end{array}$ \\
\hline 107 & Schleichera oleosa & Kusum & Whole plant & Various poojas \\
\hline 108 & Semecarpus anacardium & Bhilwa & Whole plant & During poojas \\
\hline 109 & Sesamum idicum & Til & Seed & $\begin{array}{l}\text { Shathtila Ekadashi, Makar } \\
\text { Sankranti and Til sankrarnti }\end{array}$ \\
\hline 110 & Shorea robusta & Sal & Whole plant & Worshiped as sacred plant \\
\hline 111 & Smilax zeylanica & Ramdaton & Branch & Various poojas and faste \\
\hline 112 & Solanum xanthocarpum & Bhatkatyia & Whole plant & Shiratri pooja \\
\hline 113 & Sterculia urens & Kullu & Whole plant & Kullu Deo pooja \\
\hline 114 & Syzygium cumini & Jamun & Fruit & Durga pooja \\
\hline 115 & $\begin{array}{l}\text { Tabernaemontana } \\
\text { divaricata }\end{array}$ & Chadni & Flower & Various pooja \\
\hline 116 & Tamarindus indica & Imli & Whole plant & During pooja \\
\hline 117 & Tectona grandis & Sagon & Whole plant & Worshiped as sacred plant \\
\hline 118 & Tegetes erecta & Genda & Flower & During various pooja \\
\hline 119 & Termalelia alata & Asna, Koha & Whole plant & Worshiped as sacred plant \\
\hline 120 & Terminalia arjuna & Arjun & Whole plant & Various poojas \\
\hline 121 & Terminalia chebula & Harra & Whole plant & Various poojas \\
\hline 122 & Terminallia tomentosa & Saja & Whole plant & Worshiped as sacred plant \\
\hline 123 & Thespesia lampas & Van kapas & Fruit & Various poojas \\
\hline 124 & Thevetia peruviana & pili kaner & Flower & Shivratri pooja \\
\hline 125 & Thuja orientalis & Mayurpankh & Leaves & Poojas \\
\hline 126 & Trapa bispinosa & Singhada & Seed & $\begin{array}{l}\text { Diwali \& other Poojas and to eat } \\
\text { during faste }\end{array}$ \\
\hline 127 & Woodfordia fruticosa & Dhawai & Flower & Various poojas \\
\hline 128 & Ziziphus nummularia & Ber & Fruit & $\begin{array}{l}\text { Harchhath pooja and to eat during } \\
\text { faste }\end{array}$ \\
\hline 129 & Zizyphus jujba & Ber & Branch, fruit & Hal Shasthim and Harchhath \\
\hline
\end{tabular}

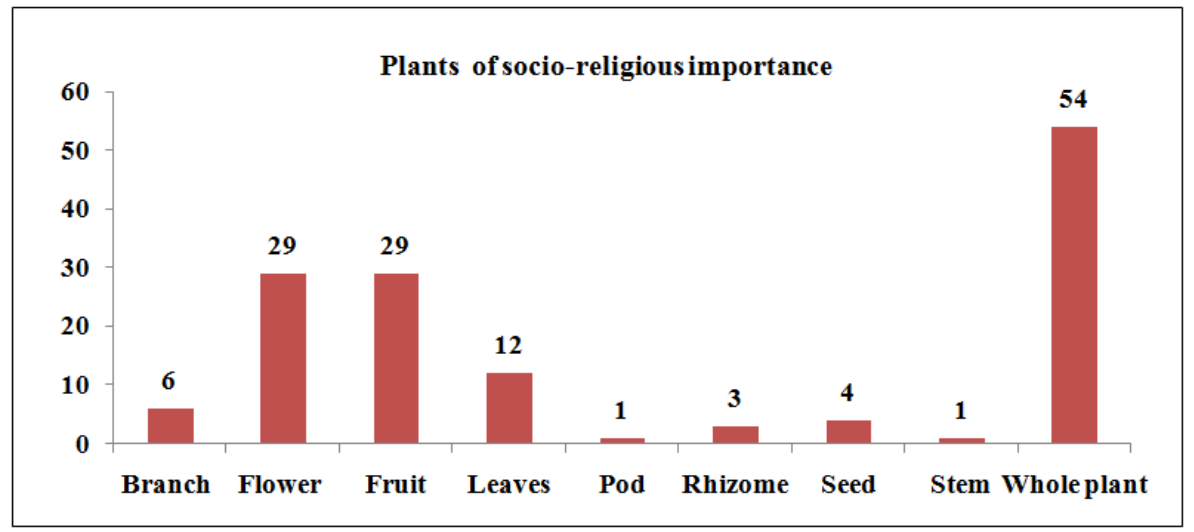

Figure 1: Plant Parts used in Religious Occations

\section{CONCLUSIONS}

It is evident from the present study that, the role of sacred groves is highly utilized as in-situ and ex-situ conservation of plant resources. Most of the plant species and their diversity are protected by the local communities for the nature conservation and socio-religious beliefs. These are the indicator of rich vegetation. One of the most important and traditional significance of sacred groves is that, it acts as a repository for various medicines and multifarious utilizable 
NTFPs, which are lacking in the surrounding forest areas. These plant species are well conserved in these graves, because

of the taboos and religious beliefs of local tribal communities. Sacred groves are also very important as a protected area to various fauna species.

\section{REFERENCES}

1. Patnaik, S. \& A. Pandey (1998) A Study of Indigenous Community Based Forest Management System: Sarna (sacred groves). In: Ramakrishnan, P. S.; K. G. Saxena \& U. M. Chandrasekara (eds.), Conserving the Sacred for Biodiversity Management, Oxford and IBH, New York, Pp:315-322.

2. Brandis, D. \& J. L. Stewart (1874) The Forest Flora of North-West and Central India.

3. Champion, H. G. \& S. K. Seth (1968) A Revised Survey of The Forest Types of India. Pp:404.

4. Forest Survey of India (2015) Indian State of Forest Report (Ministry of Environment \& Forests) Government of India. Pp:182-187.

5. Hooker, J. D. (1872-1897) The Flora of British India. 'Assisted by Various Botanists'. L. Reeve, London.

6. Mudgal, V.; K. K. Khanna \& P. K. Hajra (1997) Flora of Madhya Pradesh. Botanical Survey of India, Calcutta. vol.2, Pp:681.

7. Nair, N. C. \& A. N. Henry (1983) Flora of Tamil Nadu, India. Ser. 1: Analysis. Botanical Survey of India, Coimbatore.

8. Oommachan, M. \& J. L. Shrivastava (1996) Flora of Jabalpur. Scientific Publishers, Jodhpur. Pp: 354.

9. Patel, H. R. \& R. S. Patel (2012) Study of Sacred Groves and Sacred Plants of R.D.F. Poshina Forest Range of Sabarkantha District, North Gujrat, India. Life Sciences Leaflets, 5:11-16.

10. Singh, N. P.; K. K. Khanna; V. Mudgal \& R. D. Dixit (2001) Flora of Madhya Pradesh. Botanical Survey of India, Calcutta, vol.3, Pp:587.

11. Sinha, R. B. (2005-06 to 2014-15) Working Plan of Jabalpur Forest Division. Forest Department Madhya Pradesh Government. Part-1, Pp: 215.

12. Sukumaran, S. \& S. Jeeva (2008) A Floristic Study on Miniature Sacred Forests at Agastheeshwaram, Southern Peninsular India. Asian J. Bio. Sc. 2: 66-72.

13. Verma, D. M.; N. P. Balakrishnan \& R. D. Dixit (1993) Flora of Madhya Pradesh. Botanical Survey of India, Calcutta, vol.1, Pp:668. 
